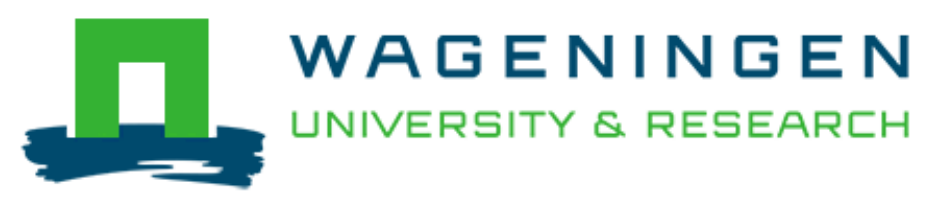

\title{
Toxicity of sediment-bound lufenuron to benthic arthropods in laboratory bioassays
}

Brock, T. C. M., Belgers, J. D. M., Boerwinkel, M. C., Jollie, L., Kraak, M. H. S., Papo, M. J., ... Roessink, I.

This is a "Post-Print" accepted manuscript, which has been published in "Aquatic Toxicology"

This version is distributed under a non-commercial no derivatives Creative Commons (ब) $(1) \Theta$ reproduction in any medium, provided the original work is properly cited and not used for commercial purposes. Further, the restriction applies that if you remix, transform, or build upon the material, you may not distribute the modified material.

Please cite this publication as follows:

Brock, T. C. M., Belgers, J. D. M., Boerwinkel, M. C., Jollie, L., Kraak, M. H. S., Papo, M. J., ... Roessink, I. (2018). Toxicity of sediment-bound lufenuron to benthic arthropods in laboratory bioassays. Aquatic Toxicology, 198, 118-128. DOI: 10.1016/j.aquatox.2018.03.005

You can download the published version at:

https://doi.org/10.1016/j. aquatox.2018.03.005 
Toxicity of sediment-bound lufenuron to benthic arthropods in laboratory bioassays

T.C.M. Brock ${ }^{a} \#$, J.D.M. Belgers, ${ }^{a}$ M-C. Boerwinkela, L. Jollie ${ }^{b}$, M.H.S. Kraak ${ }^{b}$, M.J. Papo ${ }^{b}$, J.A. Vonk ${ }^{b}$, I. Roessink ${ }^{a}$

a Wageningen Environmental Research (Alterra), Wageningen University and Research, P.O. Box 47, 6700 AA Wageningen, The Netherlands

${ }^{b}$ Institute for Biodiversity and Ecosystem Dynamics (IBED), University of Amsterdam, The Netherlands

\# Corresponding author. E-mail address: theo.brock@wur.nl (T.C.M. Brock)

Key words:

Sediment ecotoxicology

Benzoylurea insecticide

Sediment-spiked laboratory toxicity tests

Benthic macroinvertebrates

Species Sensitivity Distributions

Regulatory Acceptable Concentration 


\section{ABSTRACT}

This paper deals with species sensitivity distributions (SSDs) for the lipophilic insecticide lufenuron and benthic arthropods based on sediment-spiked laboratory toxicity tests. This compound, that inhibits chitin synthesis and moulting of arthropods, persists in sediment. Using field-collected sediment, toxicity tests were conducted with three macrocrustaceans and six insects. The Hazardous Concentration to $5 \%$ of the tested species, the HC5 (and 95\% confidence limit), derived from an SSD constructed with 10d-LC50's was $2.2(1.2-5.7) \mu \mathrm{g} / \mathrm{g}$ organic carbon (OC) in dry sediment. In addition, HC5 values derived from SSDs constructed with $28 d-$ LC10 and 28-d LC50 values were 0.13 (0.021.50) $\mu \mathrm{g} / \mathrm{g} \mathrm{OC}$ and $2.0(1.3-5.5) \mu \mathrm{g} / \mathrm{g} \mathrm{OC}$, respectively. In $28 \mathrm{~d}$ toxicity tests with Chironomus riparius and Hyalella azteca, a higher sensitivity was observed when using lufenuron-spiked field-collected sediment than in lufenuron-spiked artificial sediment. Overall, the non-biting midge $C$. riparius appeared to be a representative and sensitive standard test species to assess effects of lufenuron exposure in sediment. The Tier-1 (based on standard test species), Tier-2 (based on standard and additional test species) and Tier-3 (model ecosystem approach) regulatory acceptable concentrations (RACs) for sediment-spiked lufenuron did not differ substantially. The Tier-2 RAC was the lowest. Since to our knowledge this study is the first in the open literature that evaluates the tiered approach in the sediment effect assessment procedure for pesticides, we advocate that similar evaluations should be conducted for pesticides that differ in toxic mode-ofaction. 


\section{Introduction}

Lipophilic pesticides are frequently detected in sediments of edge-of-field freshwater ecosystems (e.g., Warren et al., 2003; Stehle and Schulz, 2015; Li et al., 2017; Wei et al., 2017), potentially leading to long-term toxic effects on benthic organisms and communities (e.g., Ding et al., 2010; Schäfer et al., 2011; MacKnight et al., 2015; Hunt et al., 2016; Moran et al. 2017). These potential ecological risks of sediment-bound pesticides were confirmed by a few micro-/mesocosm experiments conducted with spiked sediment (Boyle et al., 2016; Brock et al., 2016; Rogers et al., 2016; Yin et al., 2018). To date, prospective sediment risk assessments for pesticides are mainly based on results of laboratory bioassays with a few standard test species such as larvae of the non-biting midge Chironomus (predominantly C. dilutus and C. riparius) and the amphipod Hyalella azteca (Deneer et al. 2013). These test species are recommended in test guidelines, guidance documents and opinion papers (e.g., USEPA, 2000; OECD, 2004; ASTM, 2010; Diepens et al., 2014; EFSA, 2015; Diepens et al., 2016). It is, however, uncertain whether the derivation of a regulatory acceptable concentration in sediment (or a sediment quality standard) based on these standard benthic test species is in line with that based on a wider array of freshwater benthic organisms (e.g. species sensitivity distribution approach), as well as with that based on threshold concentrations for population-level effects observed in sediment-spiked microcosm or mesocosm tests (model ecosystem approach). In addition, in toxicity testing for sediment exposure to insecticides, predominantly pyrethroids received attention in the scientific literature (e.g., Fleming et al., 1998; Weston et al., 2009; Harwood et al., 2014; Boyle et al., 2016; Rogers et al., 2016; Li et al., 2017), while information on sediment ecotoxicology of other lipophilic insecticides is scarce.

We selected the benzoyl insecticide lufenuron as one of the benchmark substances to evaluate the prospective environmental effect assessment (ERA) procedure for sedimentassociated pesticides as proposed by the European Food Safety Authority (EFSA, 2015). 
In the European Union prospective ERA for pesticides falls under the mandate of EFSA. ERA procedures developed by EFSA for pesticides - toxicants often caharacterised by a specific toxic mode-of-action - differ from those developed for industrial chemicals by the European Chemicals Agency (ECHA, 2017). For sediment ERA, EFSA published a detailed proposal how to apply the species sensitivity distribution approach based on sedimentspiked toxicity tests with benthic arthropods and pesticides (EFSA, 2015). Lufenuron is a benzoylurea compound inhibiting chitin synthesis and moulting of arthropods (Matsumura, 2010). It is used as an insecticide and as a veterinary medicine, including uses for flea and fish lice control. According to EFSA (2008) its logKow is $5.12\left(25^{\circ} \mathrm{C}\right)$ and in water-sediment test systems its degradation half-life (DT50) ranges from 34 to 188 days. In the aquatic environment, lufenuron quickly partitions to the sediment and Brock et al. (2016) showed that in outdoor microcosms, on average $87.7 \%$ of the initial lufenuron concentration could still be detected in the sediment after 12 weeks.

Aquatic semi-field experiments and laboratory toxicity tests conducted with water-spiked (see e.g., EFSA, 2008; Brock et al., 2009 \& 2010) and sediment-spiked (Brock et al., 2016) lufenuron showed that this insecticide had direct toxic effects on aquatic arthropods (insects and crustaceans) in particular. Considering this specific toxic modeof-action, we decided to conduct additional sediment-spiked laboratory toxicity tests with a wider array of benthic arthropods to further evaluate the tiered sediment effect assessment scheme proposed for insecticides by EFSA (2015). Hence, the focus of this paper is on 10-day and 28-day sediment-spiked laboratory toxicity tests with lufenuron using standard and additional benthic test species (Tier-2 according to EFSA, 2013 \& 2015). This paper complements the study of Brock et al. (2016), that focussed on concentration-response relationships for lufenuron in sediment-spiked microcosms and in 28-day laboratory bioassays with the standard test species C. riparius, H. azteca and Lumbriculus variegatus. The aim is to present 10-day and 28-day toxicity estimates from sediment-spiked laboratory bioassays with benthic arthropods belonging to different taxonomic groups (Diptera, Ephemeroptera, Trichoptera, Megaloptera, Isopoda and 
Amphipoda) that can be used to derive a Tier- 2 regulatory acceptable concentration (RAC) for the sediment compartment. We used field-collected sediment of the same type that was used in the sediment-spiked outdoor microcosms experiment to facilitate the comparison of treatment-related responses. Furthermore, in this paper we compare the sensitivity of the standard benthic test species $\mathrm{C}$. riparius and $\mathrm{H}$. azteca between bioassays conducted with field-collected and artificial (OECD) sediment. Finally, the consistency of the tiered effect assessment scheme proposed by EFSA (2015) will be discussed using these data.

\section{Materials and methods}

\subsection{Sediment and sediment spiking}

Sediment was collected from experimental ditches at the Wageningen Environmental Research (Alterra) outdoor facility the Sinderhoeve near Renkum, the Netherlands. The ditches from which the sediment was collected were used in the past for basic ecological research (focus on nutrient transfer between water and sediments) and never treated with pesticides. The sediment was sieved through a $1 \mathrm{~mm}$ mesh sieve, mixed and stored at $-18{ }^{\circ} \mathrm{C}$. The final field-collected sediment contained $5.3 \%$ organic matter (OM) dry weight and $2.4 \%$ of the dry weight of the sediment was measured to be organic carbon (OC) (using the method described in Walinga et al., 1992). This field-collected sediment was used in sediment toxicity tests for all benthic test species (see Tables 1 and 2).

To compare the sensitivity between bioassays using field-collected and artificial sediment for the benthic standard test species $\mathrm{H}$. azteca, an additional 28-day sediment-spiked toxicity test was performed using artificial OECD sediment. This sediment was prepared in accordance to OECD (2010), having an OC content of approximately $2.5 \%$ (dry weight). Results of a 28-day sediment-spiked test with lufenuron, artificial sediment and the standard test species C. riparius reported in EFSA (2008) were provided by Syngenta. 
In our experiments, the active substance (a.s.) lufenuron was applied as the formulated product Match ${ }^{\circledR}$, as in previous semi-field experiments and laboratory sediment toxicity tests (Brock et al., 2009; Brock et al., 2016). By using Match ${ }^{\circledR}$, the plant protection product actually used in agriculture, additional organic solvent when spiking the sediment was not required.

The procedure used to prepare the dosing solutions for spiking the sediment followed Brock et al. (2016). These dosing solutions were used to obtain lufenuron concentrations ranging from 0.08 to $59.70 \mu \mathrm{g}$ a.s./g OC in dry sediment (see Tables 1 and 2 ). To spike the field-collected sediment, portions of approximately $20 \mathrm{~L}$ wet sediment were placed in concrete mixers that continuously mixed the sediment during the drip-wise application of one of the dosing solutions. The mixing of sediment continued 30 minutes postapplication. Spiking was conducted from low to high concentrations to avoid crosscontamination. The spiked sediment was stored per dose in a clean container, and mixed again for 15 min the next day. Then the batches of spiked field-collected sediments were stored in in a freezer $\left(-20^{\circ} \mathrm{C}\right)$ in portions of approximately $5 \mathrm{~L}$. Before use of these batches of sediment in the laboratory bioassays they were placed in a climate room of $20 \pm 2{ }^{\circ} \mathrm{C}$ for 7 days (ageing period).

Spiking of artificial sediment was performed in smaller volumes than those of fieldcollected sediment. For each test concentration portions of approximately $4 \mathrm{~kg}$ of wet artificial sediment (constructed following the procedure described in OECD, 2010) were dosed using a specific dosing solution (see above) and manually mixed with a handheld electric cement mixer for 10 minutes. After spiking, the artificial sediment was aged for 7 days before use in the toxicity test.

Lufenuron concentrations in the sediment were measured in subsamples of each sediment batch, to confirm dosing.

\subsection{Sediment lufenuron analysis}


To analyse the lufenuron concentrations in the sediment, the procedure described in Brock et al. (2016) was followed. Sediment concentrations and toxicity estimates were expressed in $\mu \mathrm{g}$ a.s. / g OC / kg dry sediment. The limits of detection and quantification of lufenuron in sediment were approximately 0.008 and $0.024 \mu \mathrm{g}$ a.s. / g OC / kg dry sediment, respectively. In quality control samples, the average recovery of lufenuron (n = 9) was $92 \%$ (standard deviation $13 \%$ ).

\subsection{Test species}

Since a water-spiked mesocosm experiment (Brock et al., 2009), a sediment-spiked microcosm experiment and sediment-spiked laboratory toxicity tests with the standard benthic test species C. riparius, H. azteca and L. vulgaris (Brock et al., 2016) indicated that aquatic arthropods are sensitive in particular, the benthic test species selected comprised macro-crustaceans and insects. The selection of the test species was based on their availability in laboratory cultures at Wageningen University and Research and the University of Amsterdam, and, in the case of field-collected species, their availability in sufficient numbers in nearby freshwater ecosystems (see Tables 1 and 2). An additional criterion was to perform tests with benthic arthropods belonging to different taxonomic groups (families or genera). Three taxa of the genus Chironomus were selected as test species since they often dominate the benthic arthropod community in freshwater sediments, and OECD guidelines for conducting sediment-spiked tests with these taxa are available.

The selected benthic test species comprised three macro-crustaceans (the isopod Asellus aquaticus; the amphipods Gammarus pulex and Hyalella azteca) and larvae of seven insects (the dipterans Chironomus dilutus, Chironomus riparius and Chironomus gr. thummi; the ephemeropterans Caenis horaria and Ephemera danica; the trichopteran Sericostoma personatum; the megalopteran Sialis lutaria). We are aware that C. riparius belongs to the C. gr. thummi complex. Note, however, that C. riparius used in our bioassays originated from a laboratory culture of the University of Amsterdam, while larvae C. gr. thummi were obtained from an outdoor pond at the Sinderhoeve 
experimental station (Renkum, The Netherlands). A few emerged individuals of C. gr. thummi that we obtained from the bioassay, indicated that the taxon is C. riparius. However, the sensitivity of a laboratory culture and a field population of this taxon may differ due to variation in genetic and physiological properties. Information on the origin of the test species (field collected or laboratory culture) and on the size and weight and/or life-stage of test individuals used in the 10-day and 28-day toxicity tests are presented in Tables 1 and 2, respectively. Although E. danica was originally selected for the 10-day test and S. personatum for the 28-day test, results of these experiments had to be excluded due to malfunctioning of the aeration of the test systems, resulting in mortality due to oxygen depletion.

\subsection{Sediment-spiked toxicity tests}

The 10d-LC50's for Asellus aquaticus, Gammarus pulex, Hyalella azteca, Caenis horaria, Chironomus gr. thummi and Sialis lutaria and the 28d-LC50's for G. pulex, H. azteca, C. horaria and S. lutaria, presented in this paper were obtained from tests conducted at Wageningen Environmental Research (The Netherlands). The University of Amsterdam (The Netherlands) conducted the 10d tests with Chironomus dilutus, Chironomus riparius and Sericostoma personatum, as well as the $28 \mathrm{~d}$ toxicity tests with Asellus aquaticus and Ephemera danica. Information on treatment concentrations (measured initial lufenuron concentrations in sediment), number of replicates per treatment and number of test organisms per replicate are reported in Tables 1 and 2 . The test methods used followed OECD guideline 218 (OECD, 2004) as much as possible with adaptations according to Brock et al. (2016).

In Wageningen, the sediment-spiked toxicity tests, except those with S. lutaria, were conducted in 1.5-L glass beakers containing $2 \mathrm{~cm}$ depth of sediment and $250 \mathrm{~mL}$ of Sinderhoeve spring water, with each replicate containing 10 test organisms. Before the introduction of the sediment into the test systems, dried nettle powder $(0.25 \%$ of dry sediment weight) was mixed into the sediment to provide food. In the $28 \mathrm{~d}$-tests the 
same amount of dried nettle powder was added again at day 10 to avoid food depletion (this time at the sediment surface). The test systems were placed in a climate room (temperature $18 \pm 2^{\circ} \mathrm{C} ; 16: 8 \mathrm{~h}$ light: dark regime) and were covered with a net to avoid emerged insects escaping and the water was aerated. For the insect tests, emerged individuals were routinely recorded and removed from the test system. At the end of the test the surviving animals were collected from the sediment and from the overlaying water of each test system (poured and washed through a $1 \mathrm{~mm}$ mesh sieve). For the insect test species survival was the sum of the emerged organisms and surviving larvae per test system. Sediment-spiked tests with larvae of the carnivorous insect S. lutaria were conducted individually (18-20 replicates per treatment) in $0.5 \mathrm{~L}$ glass tubes containing a sediment layer of $2 \mathrm{~cm}$ depth and $300 \mathrm{~mL}$ Sinderhoeve spring water. The test systems were placed in a climate room (temperature $18 \pm 2^{\circ} \mathrm{C} ; 16: 8 \mathrm{~h}$ light: dark regime) and were covered with a net to avoid emerged insects escaping and the water was aerated. Each individual S. lutaria larva in each replicate test system was fed with one Tubifex tubifex individual (laboratory culture) three times per week (Monday, Wednesday, Friday). At the end of the test survival of S. lutaria was recorded.

In Amsterdam, all sediment-spiked tests were conducted in aerated water-sediment test systems prepared in glass vessels (volume $300 \mathrm{~mL} ; 250 \mathrm{~mL}$ Dutch Standard Water; sediment depth $1.5 \mathrm{~cm}$ ) placed in a climate room (temperature $20 \pm 2^{\circ} \mathrm{C} ; 16: 8 \mathrm{~h}$ light: dark regime). Information on test concentrations, number of replicates per treatment and number of test animals per replicate are reported in Tables 1 and 2 . The $10 \mathrm{~d}$ toxicity tests with the chironomids $\mathrm{C}$. dilutus and C. riparius were conducted as follows. Besides mixing dried nettle powder in the sediment as food $(0.25 \%$ of the dry weight of the sediment) also $0.375 \mathrm{mg}$ of Trouvit:Tetraphyll (20:1 ratio) per chironomid larva per day was mixed in the sediment before introducing the sediment in the test vessels (also see Marinković et al., 2011). After 10 days of exposure, the experiment was finished by sieving the sediment and overlaying water from each replicate through a 300 $\mu \mathrm{m}$ sieve, and the number of surviving larvae per test vessel was recorded. In the $10 \mathrm{~d}$ 
toxicity test with S. personatum and the $28 \mathrm{~d}$ toxicity test with E. danica, the sediment was a mixture of the spiked field-collected sediment (to which $0.25 \%$ nettle powder was added as food) and quartz sand (approximately $2 \mathrm{~g}$ quartz sand per $\mathrm{g}$ field collected sediment) to provide a more stable and realistic habitat. In addition, $22 \mathrm{~g}$ of cellulose per $\mathrm{kg}$ dry sediment was added to maintain a similar ratio of OC per kg dry sediment. The lufenuron concentrations in the sediment used for the S. personatum test are reported in Table 1 and that for the E. danica test in Table 2. At the end of the 10-d toxicity tests the surviving individuals per test vessel were recorded. The $28 \mathrm{~d}$ toxicity tests with $\mathrm{A}$. aquaticus used spiked field-collected sediment to which dried nettle powder was mixed. After 28 days of exposure, the experiment was finished by sieving the sediment and overlaying water from each replicate through a $300 \mu \mathrm{m}$ sieve, and the number of surviving individuals of $A$. aquaticus per test vessel was recorded.

\subsection{Data analysis}

Based on survival data (for insects the sum of surviving larvae and emerged individuals), 10-day and 28-day LCx values (and 95\% confidence intervals) for the benthic test species were estimated using the MOSAIC web-interface for statistical analyses in ecotoxicology (Charles et al., 2017). MOSAIC is available at http://pbil.univlyon1.fr/software/mosaic/. The calculations within MOSAIC are based on the R package 'morse' (Delignette-Muller et al., 2016) and the web-interface also provides a graphical presentation of the concentration-response curves. NOECs (based on survival data) for the 10-day and 28-day toxicity tests were calculated by means of the Williams test (Williams, 1972). Calculated LCX and NOEC values were expressed in terms of initial lufenuron concentrations measured in the spiked sediment used for the tests. In laboratory toxicity tests with the standard test species (Chironomus and Hyalella) it appeared that mean exposure concentrations at the end of the toxicity tests were never lower than $80 \%$ of the initial measured concentrations, illustrating the chronic nature of 
the exposure of sediment-bound lufenuron. We assume that this is the case for all sediment-spiked toxicity tests described in this paper.

Hazardous concentrations to 5\% ( $\mathrm{HC}$ ) and 50\% (HC50) of the species tested were calculated from species sensitivity distribution (SSD) curves. These HCp values, and their $95 \%$ confidence intervals, were calculated using the MOSAIC web-interface for statistical analyses in ecotoxicology (Charles et al., 2017) and selecting the log-normal model. MOSAICSSD is able to estimate $\mathrm{HCp}$ values even if the input toxicity data are censored (Kon-Kam-King et al. 2014). We calculated the HCp values by using either LCX estimates or NOECs as input. In case the SSD was constructed with LCx values, the lower and upper limit of each toxicity estimate ( = the lower and higher value of the $95 \%$ binominal confidence intervals) was used as input. For the few species that had $a \geq L C x$ estimate, this value was used as lower limit input value in MOSAICsSD. The HCp calculations were based on the R package 'fitdistrplus' (Delignette-Muller and Dutang, 2015).

Information on CRED reporting recommendations (Moermond et al., 2016) for our study is presented in Supporting Information Table SI-1.

\section{Results}

\subsection{0-day toxicity tests}

Sediment lufenuron concentration-survival relationships and the corresponding NOEC, LC10, LC20 and LC50 values (and 95\% confidence limits) for the benthic arthropods tested in 10-day sediment-spiked bioassays (field-collected sediment) are presented in Figure 1 (see also Supporting Information Table SI-2). The relatively small difference between the $10 \mathrm{~d}-\mathrm{LC} 10,10 \mathrm{~d}-\mathrm{LC} 20$ and 10d-LC50 estimates for the Chironomus species, G. pulex and C. horaria indicate a steep concentration-response curve. The $10 \mathrm{~d}-\mathrm{LC} 50$ values showed the following order: Caenis horaria $<$ Chironomus riparius < Gammarus pulex $<$ Chironomus gr. thummi $<$ Sericostoma personatum $<$ Chironomus dilutus $<$ 
Hyallella azteca < Asellus aquaticus and Sialis lutaria. The 10d-LC50 values for the latter two species were 'greater than' values, since no effect was recorded at the highest concentration tested. The species for which $10 \mathrm{~d}$-LC50 values could be calculated generally showed a relatively narrow $95 \%$ confidence interval around the LC50; the observed ratios of the upper and lower confidence limit (= spread) ranged between 1.3 to 3.1, except for S. personatum (spread of 19.1). The broad 95\% confidence interval around the LC50 of this latter species indicates that its toxicity estimate is characterised by more uncertainty. This also explains the observation that for S. personatum the difference between the $10 \mathrm{~d}-$ NOEC and the $10 \mathrm{~d}$-LC10 is much higher than for the other test species.

\subsection{8-day toxicity tests}

Sediment lufenuron concentration-survival relationships and corresponding NOEC, LC10, LC20 and LC50 values (and 95\% confidence limits) for the 28-day sediment-spiked bioassays (field-collected sediment) with C. horaria, E. danica, S. lutaria, G. pulex and A. aquaticus are presented in Figure 2 (see also Supporting Information Table SI-3). In this figure the $28 \mathrm{~d}-$ NOEC and $28 \mathrm{~d}-\mathrm{LCX}$ values for the standard test species C. riparius and $\mathrm{H}$. azteca, tested under similar conditions as reported by Brock et al. (2016), are tabulated as well. In contrast to the 10-day toxicity test, A. aquaticus showed a clear concentration-response relationship in the 28-day bioassay, illustrating that the time-toonset of effects for this species is longer than 10 days. Again, the 28-day sedimentspiked bioassay with S. lutaria did not show a treatment-related response up to the highest sediment concentration of $27.6 \mu \mathrm{g} / \mathrm{g} \mathrm{OC}$. Most taxa that were studied in both the 10-day and 28-day tests showed lower LCx estimates in the 28-day test, except C. horaria. This latter species had a lower LC50 in the 10 -day test $(2.89 \mu \mathrm{g} / \mathrm{g} \mathrm{OC})$ than in the 28 -day test $(5.70 \mu \mathrm{g} / \mathrm{g} \mathrm{OC})$, but the $95 \%$ confidence intervals of both tests overlapped, indicating that this difference is not significant. Moreover, the field-collected test individuals used in the $10 \mathrm{~d}$ test were somewhat smaller than in the $28 \mathrm{~d}$ test (see Tables 1 and 2) and thus may have been more sensitive. For C. horaria, G. pulex and A. 
aquaticus the $95 \%$ confidence intervals of the $28 d$-LCx estimates were broader than those of the 10d-LCx estimates (see Figures 1 and 2), illustrating a higher uncertainty around the $28 \mathrm{~d}$ toxicity estimates. The $28 \mathrm{~d}-\mathrm{LC} 10$ estimates for C. horaria, E. danica and A. aquaticus in particular showed broad $95 \%$ confidence intervals, with a spread higher than 10.

The available $28 \mathrm{~d}$-LC10 estimates for the field-collected sediment showed the following order: Asellus aquaticus $<$ Chironomus riparius $<$ Caenis horaria $<$ Ephemera danica $<$ Hyalella azteca < Gammarus pulex < Sialis lutaria. For the corresponding 28d-LC50 values this order was: Chironomus riparius < Hyalella azteca $<$ Ephemera danica $<$ Gammarus pulex $<$ Caenis horaria $<$ Asellus aquaticus $<$ Sialis lutaria.

28d toxicity estimates for the standard test species C. riparius and $\mathrm{H}$. azteca using lufenuron-spiked artificial sediment are reported in Figure 3. In tests with artificial sediment a relatively steep concentration-response curve was obseved so that differences between $28 d-$ LC10 and $28 d-$ LC50 values were small. The NOECs and $28 d-$ LCX estimates derived from the bioassays using artificial sediment, however, were consistently higher when compared with similar estimates from tests using field-collected sediment. For example, C. riparius showed a $28 \mathrm{~d}-\mathrm{LC} 10$ of $4.18 \mu \mathrm{g} / \mathrm{g}$ OC using artificial sediment (Figure 3; data provided by Syngenta), compared to $0.49 \mu \mathrm{g} / \mathrm{g}$ OC with fieldcollected sediment (Figure 2; Brock et al., 2016). Similarly, these 28d-LC10 values for H. azteca were $6.20 \mu \mathrm{g} / \mathrm{g} \mathrm{OC} \mathrm{(artificial} \mathrm{sediment)} \mathrm{and} 2.83 \mu \mathrm{g} / \mathrm{g} \mathrm{OC}$ (field collected sediment), respectively.

\subsection{Species sensitivity distributions}

Species sensitivity distributions (SSDs) constructed with 28d-NOEC, 28d-LC10, 28d-LC50 and 10d-LC50 values obtained from bioassays using field-collected sediment (values reported in Figures 1 and 2) are presented in Figure 4 together with the corresponding HC5 and HC50 values and their 95\% confidence limits. In all these SSDs the standard test species C. riparius can be found in the tail of the curve, while the other standard 
benthic test species $\mathrm{H}$. azteca has a more variable position. Differences between HC5 and HC50 values are larger for SSDs constructed with 28d-LC10s (factor of 16.2) and 28dNOECs (factor of 18.0) than for SSDs constructed with 28d-LC50s (factor of 3.8) or 10dLC50s (factor of 5.5). Overall, considering the $95 \%$ confidence limits, there is a higher uncertainty surrounding the HC5 and HC50 estimates derived from SSDs constructed with LC10 and NOEC estimates, than for the HC values derived from SSDs constructed with LC50 values. Differences between HC5 values derived from SSDs constructed with $28 \mathrm{~d}-$ LC10 and $28 \mathrm{~d}-$ NOEC were relatively small. This was also the case when comparing the HC5 values derived from the $10 \mathrm{~d}-$ LC50 SSD $(2.2 \mu \mathrm{g} / \mathrm{g} \mathrm{OC})$ and $28 \mathrm{~d}$-LC50 SSDs (2.0 $\mu \mathrm{g} / \mathrm{g} \mathrm{OC})$. The HC5 values derived from the $28 \mathrm{~d}$-LC10 SSD was almost a factor of 17 lower than the HC5 derived from the 10d-LC50 SSD.

\section{Discussion}

4.1. Interpreting results of sediment-spiked toxicity tests

The bioassays with benthic arthropods described in this paper allowed addressing the combined impact of oral and dermal exposure to different fractions of lufenuron in the water-sediment test system. The high LogKow of 5.12 of lufenuron (EFSA, 2008) as well as chemical analysis in water and sediment, suggest that the majority of the spiked insecticide was not freely available in sediment pore water and overlaying water after 7 days of equilibration following spiking. This was supported by concentrations of lufenuron being below the detection limit of $0.1 \mu \mathrm{g}$ a.s./L (López-Mancisidor et al, 2008) when centrifuging and analysing the supernatant of lufenuron-spiked sediment (up to $68.6 \mu \mathrm{g}$ a.s. / g OC / kg dry sediment) four days after spiking (see Brock et al., 2016). Given the high dissipation rate of lufenuron from water to sediment and its persistence in this compartment, as well as the difficulties in accurately quantifying low lufenuron concentrations in (pore) water, we used measured initial concentrations in the total sediment to express the LCX and NOEC estimates. 
To facilitate a comparison of toxicity estimates with existing data in the published literature, sediment concentrations and toxicity estimates were expressed in $\mu \mathrm{g}$ a.s. $/ \mathrm{g}$ OC / kg dry sediment. Nevertheless, bioavailability of sediment-associated pesticides is reported to be influenced to some extent by other sediment properties than OC content, such as sediment grain size, clay content and type of organic matter present (e.g., Fleming et al. 1998; Palmquist et al., 2011; Li et al., 2017). In addition, a decline in bioavailability of persistent chemicals due to ageing processes is reported in the scientific literature (e.g. Landrum et al., 1992; Alexander, 2000; Choi et al., 2012; Nybom et al., 2015). Since a 7-day equilibrium phase after spiking was used in all toxicity tests described in the present paper, the possible decline in bioavailability due to ageing processes was most likely similar in all tests.

Given the information above, a possible lower bioavailability of sediment-associated lufenuron in artificial sediment than in field-collected sediment may have contributed to the overall higher $28 \mathrm{~d}-\mathrm{LCx}$ values for $\mathrm{C}$. riparius and $\mathrm{H}$. azteca in tests that used artificial sediment. In addition, the organic matter (and associated lufenuron) occurring in the spiked field-collected sediment might have been a more preferred food than the organic matter (peat) included in the spiked artificial OECD sediment. If in tests with artificial sediment the test organisms consumed more of the additional food supplied (e.g., Trouvit:Tetraphyll and/or dried nettle powder that was not spiked) and less of the organic matter present in the spiked sediment, then oral exposure was likely lower as well. Another explanation of the overall higher sensitivity of $\mathrm{C}$. riparius and $\mathrm{H}$. azteca in tests with field-collected sediment might be the more optimal growing conditions for the test organisms (e.g. due to a more diverse microbial community as food source) compared to the tests with artificial sediment. Toxic effects of chitin synthesis inhibitors such as lufenuron become apparent during the sensitive life stages of moulting and metamorphosis (e.g. Tunaz and Uygun, 2004), and these processes may occur at higher rates under more optimal growth conditions. It cannot be excluded, however, that some 
confounding factors (e.g. natural toxicants such as sulphites) were present in the fieldcollected sediment that enhanced the effects of lufenuron.

\subsection{Differences in sensitivity between benthic test species}

In the present study, differences in sensitivity between benthic test species may, at least in part, be explained by species-specific differences in sediment-dwelling behaviour and bioturbation activity, affecting exposure to, and uptake of, lufenuron. For example, epibenthic test species (e.g. A. aquaticus and G. pulex) were predominantly at the sediment-water interface of the test systems, in contrast to the endo-benthic arthropods (e.g. chironomids and C. horaria) that were pre-dominantly found buried in the sediment of the test system, affecting dermal exposure. In addition, differences in feeding habit between species may have caused differences in oral exposure. For example, species like A. aquaticus and G. pulex are shredders that usually feed on larger particulate organic matter, while species like chironomids feed on smaller particles. Furthermore, macrocrustaceans are reported to be more active bioturbators than chironomids, affecting dermal and oral exposure to toxicants and, consequently, species-specific sensitivity (e.g. Van der Meer et al., 2017). Therefore, the observed differences in LCx values between benthic arthropods may not only be explained by intrinsic differences in sensitivity, but also by species-specific differences in exposure and autecological characteristics, despite the fact that we used the same type of homogenised fieldcollected sediment in most tests. The test results reported in this paper and in Brock et al. (2016), however, support the observation of Maltby et al. (2005) that taxonomy plays a larger role in explaining sensitivity to insecticides than habitat. In 28 -day sedimentspiked toxicity tests with lufenuron, all benthic arthropods tested, except perhaps S. lutaria, were more than an order of magnitude more sensitive than the oligochaete worm Lumbriculus variegatus (28d-EC50 of 213 - $1099 \mu \mathrm{g}$ a.s./g OC; Brock et al., 2016).

The observation that in the 10-day toxicity tests clear concentration-response relationships could not be observed for $\mathrm{A}$. aquaticus and $\mathrm{S}$. lutaria might be due to the fact that effects of lufenuron become apparent only when these species moult, which 
might not have occurred in these relatively short tests. The fact that $\mathrm{S}$. lutaria is a semivoltine aquatic insect with a life-cycle that covers two years (Elliot, 1996) might also explain why we did not observe a concentration-response relationship in the 28-day toxicity tests. It is well possible that during the test the larvae of S. lutaria did not develop into other instars and that moulting did not occur. Therefore, we suggest that in conducting sediment-spiked toxicity tests with an insect growth-regulator like lufenuron, not only the sensitive benthic life stage should be tested, but also that the test should be long enough to include the critical stages of moulting and any metamorphosis. However, these requirements may be difficult to achieve in laboratory bioassays with field-collected benthic taxa characterised by a long life-cycle and slow growth. Furthermore, to explain differences in concentration-response relationships for lufenuron between different benthic arthropods in sediment-spiked toxicity tests, information on the dynamics of body burdens and the toxicokinetics and toxicodynamics of lufenuron in the different test species may be helpful (e.g. Escher et al., 2011).

In the present study, we conducted sediment-spiked toxicity tests with lufenuron and ten different benthic arthropod species, including two amphipods, one isopod, three chironomids, two ephemeropterans, one trichopteran and one megalopteran. Other freshwater arthropods sporadically used in published sediment-spiked toxicity tests with pesticides comprise larvae of the mayflies Jappa kutera (Leonard et al., 2001), Ephoron virgo (De Haas et al., 2005), Hexagenia limbata and H. bilineata (e.g. Harwood et al., 2014). The literature review of Deneer et al. (2013) showed that a limited number of benthic arthropods are routinely used as test organisms in sediment-spiked toxicity tests with insecticides. The vast majority of sediment-spiked toxicity tests are conducted using the midge Chironomus sp. and the amphipod H. azteca.

The present study is the first published in the open literature that deals with sedimentspiked toxicity tests conducted with an insecticide using sufficient benthic arthropod species to allow an SSD approach in the sediment effect assessment procedure as proposed by EFSA (2015). SSDs constructed with toxicity data derived from sediment- 
spiked bioassays and benthic invertebrates belonging to various taxonomic groups have so far only been published for copper (Roman et al., 2007; Van der Meer, 2017), nickel (Besser et al., 2013) and the biocide tributyltin (Diepens et al., 2017).

In the open literature only a few other toxicity data for lufenuron and benthic arthropods can be found. In the dossier for lufenuron, EFSA (2008) reported a 28-d NOEC for C. riparius of $0.04 \mathrm{mg}$ a.s. $/ \mathrm{kg}$ dry sediment which approximately equals $2.35 \mu \mathrm{g}$ a.s./g OC (using an OC content of $1.7 \%$ in the artificial sediment used, based on personal communication with Mick Hamer of Syngenta). The underlying data for this NOEC were provided to us by Syngenta and re-analysed (see Figure 3). Hooper et al. (2005) published a 10d-NOEC (endpoint survival) for C. riparius of $0.06 \mathrm{mg}$ a.s. $/ \mathrm{kg}(=1.33 \mu \mathrm{g}$ a.s./g OC), while in our toxicity tests this 10d-NOEC was somewhat higher $(3.32 \mu \mathrm{g}$ a.s./g OC). Deneer et al. (2013) reported a $21 \mathrm{~d}$-LC50 for A. aquaticus of $6.59 \mu \mathrm{g}$ a.s./g OC, while in our experiments the 10d-LC50 and 28d-LC50 values for this species were $>31.7 \mu \mathrm{g}$ a.s./g OC and $10.92 \mu \mathrm{g}$ a.s./g OC, respectively. Thus, the toxicity data for benthic arthropods and lufenuron available in the open literature are more or less in line with our observations.

4.3. Calibration of the EFSA effect assessment approaches for sediment organisms The results of the sediment-spiked bioassays with lufenuron presented in this paper can be used to evaluate the lower tier effect assessment approaches in prospective sediment ERA, such as the standard test species approach (Tier-1) and the SSD approach (Tier-2). EFSA (2015) proposed to use in first instance the chronic regulatory acceptable concentration for pelagic organisms ( $\left.=\mathrm{RAC}_{\mathrm{sw} ; \mathrm{ch}}\right)$ and the equilibrium partitioning (EqP) approach as an initial screening to assess the risk of sediment exposure to benthic organisms, but to apply an extrapolation factor of 10 to cover the possibility of exposure due to sediment ingestion (Tier-0, modified EqP approach). According to EFSA (2015) this Tier-0 RAC sed; EqP (expressed in terms of concentration per g OC in dry sediment) can be derived with the following formula: $\left(\mathrm{RAC}_{\mathrm{sw} ; \mathrm{ch}} * \mathrm{~K}_{\mathrm{oc}}\right) / 10$. Using the information of the Pesticides Properties Data Base (https://sitem.herts.ac.uk/aeru/ppdb/en/atoz.htm ) the 
$\mathrm{RAC}_{\text {sw; ch }}$ for lufenuron can be derived by selecting the lowest chronic NOEC/EC10 value available for pelagic Tier-1 test species ( $=21-\mathrm{d}$ NOEC for Daphnia magna of $0.1 \mu \mathrm{g} / \mathrm{L}$ ) and by applying an assessment factor $(A F)$ of $10\left(R^{2} C_{s w ; c h}=0.01 \mu \mathrm{g} / \mathrm{L}\right)$. Selecting the $K_{\text {foc }}$ value of $41182 \mathrm{~L} / \mathrm{kg} \mathrm{OC}$ reported for lufenuron in this database as a proxi of the $\mathrm{K}_{\mathrm{oc}}$ the Tier- 0 RAC sed; $E_{\text {gP }}=(0.01 * 41182) / 10=41.182 \mu \mathrm{g}$ a.s. $/ \mathrm{kg} \mathrm{OC}=0.041 \mu \mathrm{g}$ a.s. $/ \mathrm{g} \mathrm{OC}$ (Figure 5).

In prospective Tier- 1 tests artificial sediment is usually used (e.g. OECD, 2004) and in the dossier for lufenuron the $28 \mathrm{~d}$ NOEC value of $\mathrm{C}$. riparius is selected for sediment risk assessment (EFSA, 2008). EFSA (2015) proposed to use C. riparius and H. azteca as Tier-1 benthic test species and to apply an AF of 10 to the lowest chronic NOEC/EC10 value. From the data presented in Figure 3 it appears that C. riparius was more sensitive than H. azteca. Selecting the $28 \mathrm{~d}-$ NOEC of $2.35 \mu \mathrm{g}$ a.s./g OC for C. riparius and the application of an AF of 10 results in a Tier-1 RAC sed of $0.235 \mu \mathrm{g}$ a.s./g OC (Figure 5). According to EFSA (2015), a Tier-2 RAC sed can be derived by applying the SSD approach by either selecting the HC5 value from an SSD constructed with $10 \mathrm{~d}$-LC50 data (sediment-spiked toxicity tests) and the application of an AF of 15, or the HC5 value from an SSD constructed with chronic NOEC/EC10 data and the application of an AF of 3. Both SSDs, however, should be constructed with toxicity data for at least 8 different benthic arthropod species. This latter criterion was only met for the 10d-LC50 SSD. Selecting the HC5 of the $10 \mathrm{~d}$-LC50 SSD (=2.2 $\mu \mathrm{g}$ a.s./g OC) and the application of an AF of 15 results in a Tier-2 RAC $_{\text {sed }}$ of $0.147 \mu$ a.s./g OC (Figure 5). It could be argued that C. riparius (laboratory culture) and C. gr. thummi (field population) in fact are the same taxon and that they should not both be used in the SSD. Constructing the 10d-LC50 SSD without the toxicity estimate for C. gr. thummi, however, results in a more or less similar HC5 of $2.0(1.1-6.2) \mu \mathrm{g}$ a.s./g OC, and consequently hardly affects the derived Tier-2 $\mathrm{RAC}_{\text {sed. }}$ Selecting the HC5 from the SSD constructed with seven $28 \mathrm{~d}-\mathrm{LC} 10$ values $(0.13$ $\mu \mathrm{g}$ a.s./g OC) or that from the corresponding $28 \mathrm{~d}-\mathrm{NOEC}$ SSD $(0.10 \mu \mathrm{g}$ a.s./g OC) and an application of an AF of 3 would result in a provisional $\mathrm{RAC}_{\text {sed }}$ of $0.033-0.043 \mu \mathrm{g}$ a.s./g 
OC. Note, however, that the EFSA criterion of toxicity data for at least 8 arthropod taxa was not met for the SSD constructed with $28 d$ toxicity data. In addition, the HC5 values derived from $28 \mathrm{~d}$-LC10/NOECs are less reliable considering the high uncertainties associated with these HC5 values as indicated by their very broad confidence limits (see Figure 4). Since for less than 8 different arthropod taxa $28 d$ toxicity estimates are available, the Geometric mean approach is an alternative option for $\mathrm{RAC}_{\text {sed }}$ derivation since the measurement endpoint in all tests was survival and toxicity estimates are available for insects and crustaceans (see EFSA, $2013 \& 2015$ ). The geometric mean 28d LC10 values for the insects C. riparius, C. horaria and E. danica, and the crustaceans H. azteca, G. pulex and A. aquaticus (see Figure 2) were 1.021 and $1.024 \mu \mathrm{g}$ a.s./g OC, respectively. Note that the corresponding greater than value for the insect $\mathrm{S}$. lutaria was not included in this calculation. Applying and AF of 10 to the lowest geometric mean $28 \mathrm{~d}$ LC10 value of $1.021 \mu \mathrm{g}$ a.s./g OC for insects, results in a Tier-2 RAC sed of $0.1021 \mu \mathrm{g}$ a.s./g OC (Figure 5).

According to EFSA (2013; 2015) a Tier-3 RAC sed might be derived from an appropriate micro-/mesocosm experiment by selecting the sediment concentration of the highest treatment-level that did not result in population-level effects on benthic organisms and the application of an AF of 2. The NOEC of the most sensitive benthic populations (Tanytarsini and Chironomini) in the sediment-spiked microcosm experiment reported by Brock et al. (2016) was $0.79 \mu$ a.s./g OC. In addition, also a NOEC of $0.79 \mu \mathrm{g}$ a.s./g OC for the benthic community was observed in this microcosm experiment. Applying an AF of 2 results in a Tier- 3 RAC $_{\text {sed }}$ of $0.395 \mu \mathrm{g}$ a.s./g OC (Figure 5).

A tiered effect assessment approach is consistent if lower tiers are more conservative than higher tiers. Indeed, the Tier-0 screening method based on equilibrium partitioning resulted in the lowest $\mathrm{RAC}_{\text {sed, }}$ and the two Tiers based on sediment-spiked laboratory bioassays were lower than the Tier- $3 \mathrm{RAC}_{\text {sed }}$ derived from the sediment-spiked microcosm experiment (Figure 5). However, the Tier-2 RACs based on the SSD approach and the Geometric mean approach are lower than the Tier-1 RAC based on standard test 
species. Furthermore, it appears that differences between the Tier-1, Tier-2 and Tier-3 RAC $_{\text {sed }}$ values presented in Figure 5 are relatively small. This, however, may at least in part be explained by the fact that lufenuron persisted in the sediment compartment of all test systems used, causing a similar long-term exposure regime in the laboratory bioassays and in the outdoor microcosms.

\subsection{Concluding remarks}

In conclusion, the sediment-spiked toxicity data presented in this study suggest that for any sediment-associated contaminant it will be quite an effort to obtain $28 \mathrm{~d}$-LC10/EC10 values with acceptable confidence limits when selecting non-standard benthic test species. Toxicity data characterised by a low ratio of the upper and lower confidence limit (e.g. a spread less than 10) were more easily obtained for the $28 d-$ LC50 estimates and in the $10-d$ sediment-spiked toxicity tests. It may, therefore, be more appropriate to develop effect assessment schemes for RAC sed derivation based on 10d-LC50 or 28d$\mathrm{L}(\mathrm{E}) \mathrm{C50}$ data. For lufenuron, that persisted in the sediment, the Tier-0 approach proposed by EFSA (2015), based on equilibrium partitioning and an additional extrapolation factor of 10 to cover dietary exposure, seems to be a cost-effective screening method to avoid laborious toxicity testing. An extrapolation factor seems to be necessary in the case of lufenuron, since without this the Tier-0 RAC sed; EqP would become higher that the RACsed derived in all other tiers. The toxicity data for benthic arthropods and lufenuron presented in this paper reveal that C. riparius in particular is a representative and sensitive standard test species. From a cost-effective point of view, and considering the Tier- 1 to Tier- 3 RAC $_{\text {sed }}$ values presented in Figure 5, the added value of conducting sediment-spiked toxicity tests with a wider array of benthic tests species appeared to be limited. Yet, in order to evaluate the general consistency of the prospective effect assessment approaches for sediment-bound pesticides, the prospective effect assessment methods proposed by EFSA (2015) and Diepens et al. (2016) need to be explored for more substances that persist in sediments and that differ in toxic modeof-action. 
Acknowledgements. The present study received financial support of the Dutch Ministry of LNV (project BO-20-002-001). The authors are indebted to Laura Buyse, Arrienne Matser and Steven Crum for their help in analysing lufenuron in sediment samples. Mick Hamer critically read a previous version of the paper and made valuable suggestions for improvement.

\section{References}

Alexander, M., 2000. Aging, bioavailability, and overestimation of risk from environmental pollutants. Environmental Science \& Technology 34, 4259-4265.

ASTM (American Society for Testing and Materials), 2010. E1706-05, Standard Test Method for Measuring the Toxicity of Sediment-Associated Contaminants with Freshwater Invertebrates. ASTM International, West Conshohocken, PA, USA.

Besser, J.M., Brumbaugh, W.G., Ingersoll, C.G., Ivey, C.D., Kunz, J.L., Kemble, N.E., Schlekat, C.E., Rogevick Garman, E., 2013. Chronic toxicity of nickel-spiked freshwater sediments: variation in toxicity among eight invertebrate taxa and eight sediments. Environmental Toxicology and Chemistry 32, 2495-2506.

Boyle, R.L., Hoak, M.N., Pettigrove, V.J., Hoffmann, A.A., Long, S.M., 2016. Comparing the impacts of sediment-bound bifenthrin on aquatic macroinvertebrates in laboratory bioassays and field microcosms. Ecotoxicology \& Environmental Safety 133, 489-500.

Brock, T.C.M., Roessink, I., Belgers, J.D.M., Bransen, F., Maund, S.J., 2009. Impact of a benzoyl urea insecticide on aquatic macroinvertebrates in ditch mesocosms with and without non-sprayed sections. Environmental Toxicology and Chemistry 28, 2191-2205.

Brock, T.C.M., Belgers, J.D.M., Roessink, I., Cuppen, J.G.M., Maund, S.J., 2010. Macroinvertebrate responses to insecticide application between sprayed and adjacent nonsprayed ditch sections of different sizes. Environmental Toxicology and Chemistry 29, 1994-2008. 
Brock, T.C.M., Bas, D.A., Belgers, J.D.M., Bibbe, L., Boerwinkel, M-C., Crum, S.J.H., Diepens, N.J., Kraak, M.H.S., Vonk, J.A., Roessink, I., 2016. Effects of sediment-spiked lufenuron on benthic macroinvertebrates in outdoor microcosms and single-species toxicity tests. Aquatic Toxicology 177, 464-475.

Charles, C., Veber, P., Delignette-Muller, M.L., 2017. MOSAIC: a web-interface for statistical analyses in ecotoxicology. Environmental Science and Pollution Research. DOI $10.1007 / \mathrm{s} 11356-017-9809-4$

Choi, H., Nfodzo, P.A., Lawal, W.A., Al-Abed, S.R., Seo, Y., 2012. Phenomenological and spectroscopic analysis on the effects of sediment ageing and organic carbon on the fate of a PCB congener spiked to sediment. J ournal of Hazardous Materials 239-240, 325-332.

De Haas, E.M., Roessink, I., Verbree, B., Koelmans, A.A., Kraak, M.H.S., Admiraal, W., 2005. Influence of sediment quality on the responses of benthic invertebrates after treatment with the fungicide triphenyltin acetate. Environmental Toxicology and Chemistry 24, 1133-1139.

Delignette-Muller, M.L., Dutang, C., 2015. Fitditrplus: an R package for fitting distributions. J ournal of Statistical Software 64,1-34. http://www.jstasoft.org/v64/i04/.

Delignette-Muller, M.L., Ruiz,P., Charles, S., Duchemin, W., Lopes, C., Kon-Kam-King, G., Veber, P., 2016. Morse: modelling tools for reproduction and survival data in ecotoxicology. https://CRAN.R-project.org/package=morse. $\mathrm{R}$ package version 2.2.0

Deneer, J.W., Arts, G.H.P., Brock, T.C.M., 2013. Sediment toxicity data for benthic organisms and plant protection products. A literature review. Alterra report 2485, Wageningen, The Netherlands, $47 \mathrm{pp}$

Diepens, N.J., Arts, G.H.P., Brock, T.C.M., Smidt, H., Van den Brink, P.J., Van den HeuvelGreve, M.J., Koelmans, A.A., 2014. Sediment toxicity testing of organic chemicals in the context of prospective risk assessment: A review. Critical Reviews in Environmental Science and Technology 44, 255-302. 
Diepens, N.J., Koelmans, A.A., Baveco, H., Van den Brink, P.J., Van den Heuvel-Greve, M.J., Brock, T.C.M., 2016. Prospective environmental risk assessment for sediment-bound organic chemicals: A proposal for tiered effect assessment. Reviews of Environmental Contamination and Toxicology 239, 1-77.

Ding, Y., Harwood, A.D., Foslund, H.M., Lydy, M.J., 2010. Distribution and toxicity of sediment-associated pesticides in urban and agricultural waterways from Illinois, USA. Environmental Toxicology and Chemistry 29, 149-157.

ECHA (European Chemicals Agency), 2017. Guidance on Information Requirements and Chemical Safety Assessment, Chapter R.7b Endpoint specific guidance. ECHA-17-G-10-EN, DOI: $10.2823 / 84188$.

EFSA (European Food Safety Authority), 2008. Conclusion on the peer review of lufenuron. EFSA Scientific Report 189, 1-130.

EFSA (European Food Safety Authority), 2013. Guidance on tiered risk assessment for plant protection products for aquatic organisms in edge-of-field surface waters. EFSA Journal 11(7):3290, 268 pp.

EFSA (European Food Safety Authority), 2015. Scientific opinion on the effect assessment for pesticides on sediment organisms in edge-of-field surface water. EFSA Journal 13(7): 4176, $145 \mathrm{pp}$.

Elliot, J.M., 1996. British freshwater Megaloptera and Neuroptera: A key with ecological notes. Freshwater Biological Association Scientific Publication No. 54. Cumbria (UK): The Ferry House, Ambleside, 75 pp.

Escher, B.I., Ashauer, R., Dyer, S., Hermens, J.L., Lee, J-L., Leslie, H.A., Mayer, P., Meador, J.P., Warne, M.S., 2011. Crucial role in mechanisms and modes of toxic action for understanding tissue residue toxicity and internal effect concentrations of organic chemicals. Integrated Environmental Assessment and Management 7, 28-49. 
Fleming, R.J., Holmes, D., Nixon, S.J., 1998. Toxicity of permethrin to Chironomus riparius in artificial and natural sediment. Environmental Toxicology and Chemistry 17, 1332-1337.

Harwood, A., Rothert, A.K., Lydy, M.J. (2014). Using Hexagenia in sediment bioassays: methods, applicability, and relative sensitivity. Environmental Toxicology and Chemistry $33,868-874$.

Hooper, H.L., Sibly, R.M., Hutchinson, T.H., Maund, S.J., 2005. Joint effect of density and a growth inhibitor on the life history and population growth rate of the midge Chironomus riparius. Environmental Toxicology and Chemistry 24, 1140-1145.

Hunt, L., Bonetto, C., Resh, V.H., Forsin Buss, D., Fanelli, S., Marrochi, N., Lydy, M.J., 2016. Insecticide concentrations in stream sediments of soy production regions of South America. Science of the Total Environment 547, 114-124.

Kon-Kam-King, G., Veber, P., Charles, S., Delignette-Muller, M.L., 2014. MOSAIC_SSD: a new web tool for species sensitivity distribution to include cencered data by maximum likelyhood. Environmental Toxicology and Chemistry 33, 2133-2139.

Landrum, P.F., Eadie, B.J., Faust, W.R., 1992. Variation in the bioavailability of polycyclic aromatic hydrocarbons to the amphipod Diporeira (spp.) with sediment aging. Environmental Toxicology and Chemistry 11, 1197-1208.

Leonard, A.W., Hyne, R.V., Lim, R.P., Leigh, K.A., Le, J., Beckett, R., 2001. Fate and toxicity of endosulfan in Namoi river water and bottom sediment. J ournal of Environmental Quality 30, 750-759.

Li, H., Cheng, F., Wei, Y., Lydy, M.L., You, J. (2017). Global occurrence of pyrethroid insecticides in sediment and the associated toxicological effects on benthic invertebrates: An overview. J ournal of Hazardous Materials 324, 258-271.

López-Mancisidor, P., Van den Brink, P.J., Crum, S.J.H., Maund, S.J., Carbonell, G., Brock, T.C.M., 2008. Responses of zooplankton in lufenuron-stressed experimental ditches in the 
presence or absence of uncontaminated refuges. Environmental Toxicology and Chemistry 27, 1317-1331.

Maltby, L., Blake, N., Brock, T.C.M., Van den Brink, P.J., 2005. Insecticide species sensitivity distributions: Importance of test species selection and relevance to aquatic ecosystems. Environmental Toxicology and Chemistry 24, 379-388.

Marinković, M., Verweij, R.A., Nummerdor, G.A., Jonker, M.J., Kraak, M.H.S., Admiraal, W., 2011. Life cycle responses of the midge Chironomus riparius to compounds with different modes of action. Environmental Science \& Technology 45, 1645-1651.

Matsumura, F., 2010. Studies on the action mechanism of benzoylurea insecticides to inhibit the process of chitin synthesis in insects: A review on the status of research activities in the past, the present and the future prospects. Pesticide Biochemistry and Physiology 97, 133-139.

McKnight, U.S., Rasmussen, J.J., Kronvang, B., Binning, P.J., Bjerg, P.L., 2015. Sources, occurrence and predicted aquatic impact of legacy and contemporary pesticides in streams. Environmental Pollution 200, 64-76.

Moermond, C.T.A., Kase, R., Korkaric, M., Ågerstrand, M., 2016. CRED: Criteria for reporting and evaluating ecotoxicity data. Environmental Toxicology and Chemistry 35, 1297-1309.

Moran, P.W., Nowell, L.H., Kemble, N.E., Mahler, B.J., Waute, I.R., Van Metre, P.C., 2017. Influence of sediment toxicity on macroinvertebrate communities across 99 wadable streams of the Midwestern USA. Science of the Total Environment 599-600, 1469-1478.

Nybom,I., Waissi-Leinonen, G., Mäenpää, K., Leppänen, M.T., Kukkonen, J.V.K., Werner, D., Akkanen, J., 2015. Effects of activated carbon ageing in three PCB contaminated sediments: Sorption efficiency and secondary effects on Lumbriculus variegatus. Water Research 85, 413-421 
OECD, 2004. Guidelines for testing of chemicals - 218: Sediment-Water Chironomid Toxicity Test Using Spiked Sediment. OECD Publishing, Paris.

OECD, 2010. Test No. 233: Water Chironomid Life-Cycle Toxicity Test Using Spiked Water or Spiked Sediment. OECD Publishing, Paris.

Palmquist, K., Fairbrother, A., Salatas, J, Guiney, P.D., 2011. Environmental fate of pyrethroids in urban and suburban stream sediments and the appropriateness of Hyalella azteca model in determining ecological risk. Integrated Environmental Assessment and Management 7, 325-335.

Rogers, H.A., Schmidt, T.S., Dabney, B.L., Hladik, M.L., Mahler, B.J., Van Metre, P.C., 2016. Bifenthrin causes trophic cascade and altered insect emergence in mesocosms: Implications for small streams. Environmental Science \& Technology 50, 11974-11983.

Roman, Y.E., De Schamphelaere, A.C., Nguyen, L.T.H., Janssen, C.R., 2007. Chronic toxicity of copper to five benthic invertebrates in laboratory-formulated sediment: Sensitivity comparison and preliminary risk assessment. Science of the Total Environment 387, 128-140.

Schäfer, R.B., Pettigrove, V., Rose, G., Allinson, G., Wightwick, A., Von der Ohe, P.C., Shimeta, J., Kühne, R., Kefford, B.J., 2011. Effects of pesticides monitored with three sampling methods in 24 sites on macroinvertebrates and microorganisms. Environmental Science \& Technology 45, 1665-1672.

Stehle S., Schulz, R., 2015. Agricultural insecticides threaten surface waters at the global scale. Proc. Natl. Acad. Sci. USA, 112:5750-5755.

Tunaz, H., Uygun, N., 2004. Insect growth regulators for insect pest control, Turkish Journal of Agriculture and Forestry 28, 377-387.

USEPA (US Environmental Protection Agency), 2000. Methods for measuring the toxicity and bioaccumulation of sediment-associated contaminants wit freshwater invertebrates, 
$2^{\text {nd }}$ ed. EPA 600/R-99/064. Office of Research and Development, Office of Science and Technology, Washington, DC., USA.

Van der Meer, T.V., de Baat, M.L., Verdonschot, P.F.M., Kraak, M.H.S., 2017. Benthic invertebrate bioturbation activity determines species specific sensitivity to sediment contamination. Frontiers in Environmental Science, 5, article 83. Doi: 10.3389/fenvs.2017.00083.

Walinga, I., Kithome, M., Novozamsky, I., Houba, V.J.G., Van der Lee, J.J., 1992. Spectrophotometric determination of organic carbon in soil. Communications in Soil Science and Plant Analysis 23, 1935-1944.

Warren, N., Alan, I.J., Carter, J.E., House, W.A., Parker, A., 2003. Pesticides and other micro-organic contaminants in freshwater sedimentary environments - a review. Applied Geochemistry 18, 159-194.

Wei, Y., Li, H., Zhang, J., Xiong, J., Yi, X., You, J., 2017. Legacy and current-use insecticides in agricultural sediments from South China: Impact of application pattern on occurrence and risk. J ournal of Agricultural and Food Chemistry 65, 4247-4254.

Weston, D.P., You, J., Harwood, A.D., Lydy, M.J., 2009. Whole sediment toxicity identification evaluation tools for pyrethroid insecticides: III. Temperature manipulation. Environmental Toxicology and Chemistry 28, 173-180.

Williams, D.A., 1972. The comparison of several dose levels with a zero dose control. Biometrics 28, 519-531.

Yin, X.H., Brock, T.C.M., Barone, L.E., Belgers, J.D.M., Boerwinkel, M-C., Buijse, L., Van Wijngaarden, P.P.A., Hamer, M., Roessink, I., 2018. Exposure and effects of sedimentspiked fludioxonil on macroinvertebrates and zooplankton in outdoor aquatic microcosms. Science of the Total Environment 610-611, 1222-1238. 

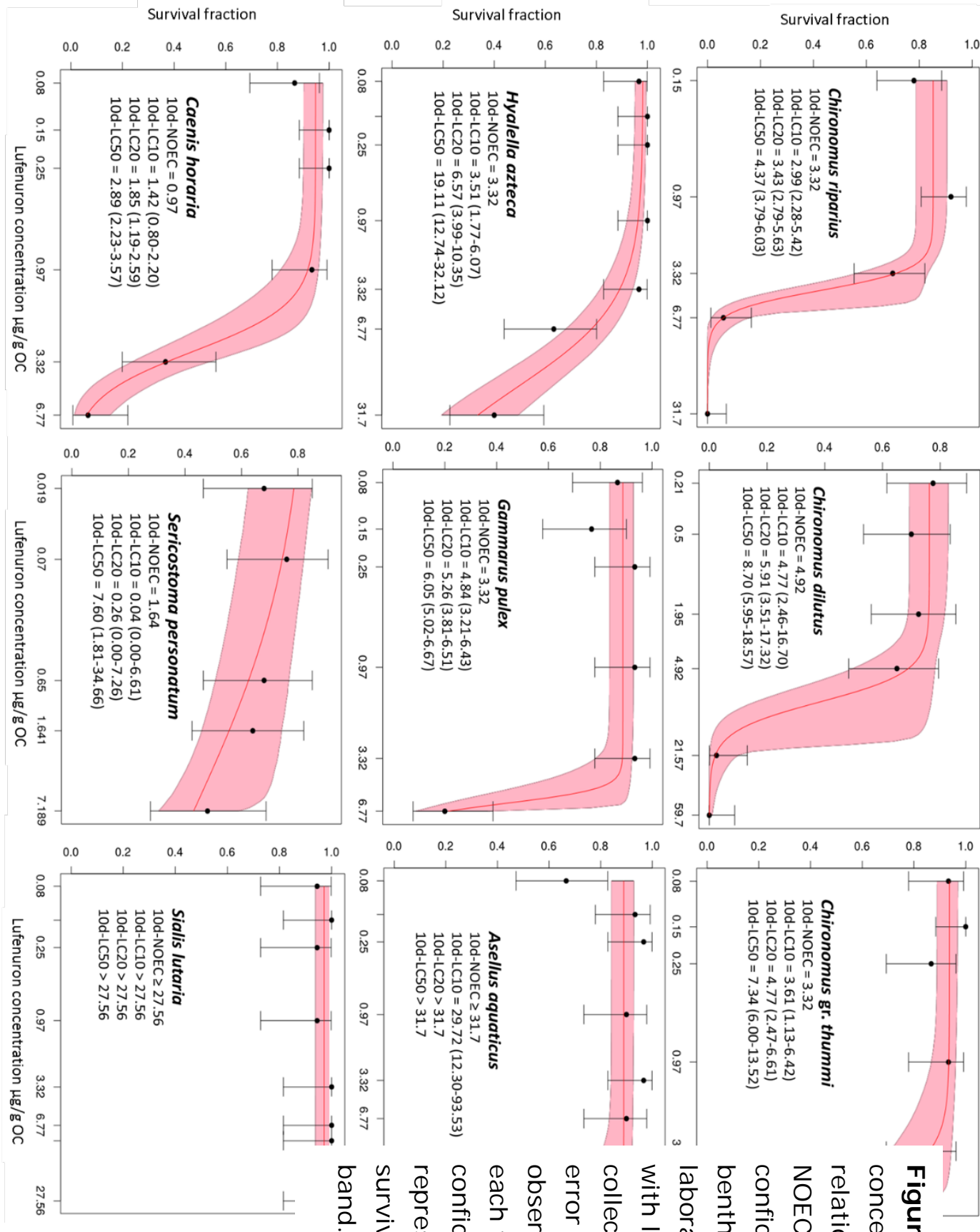

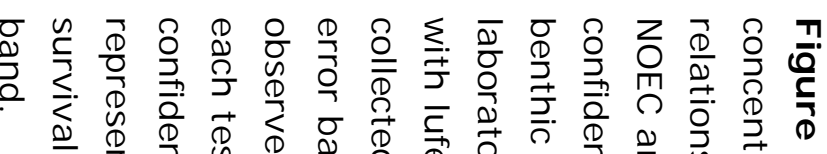

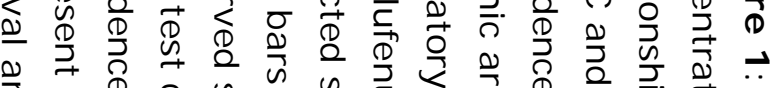

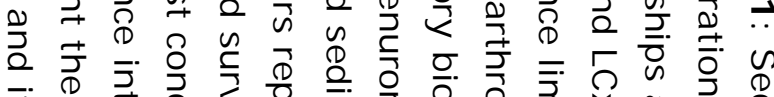

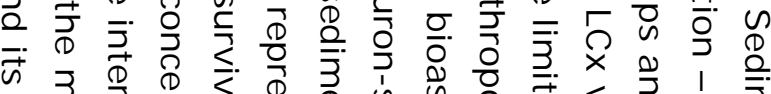

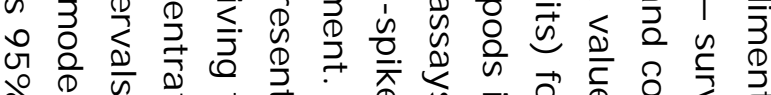

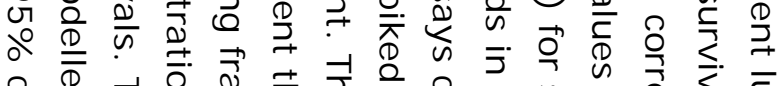

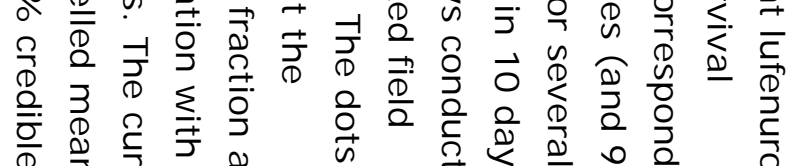

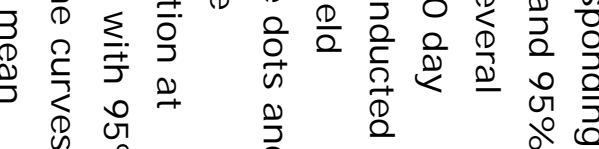
능 응 


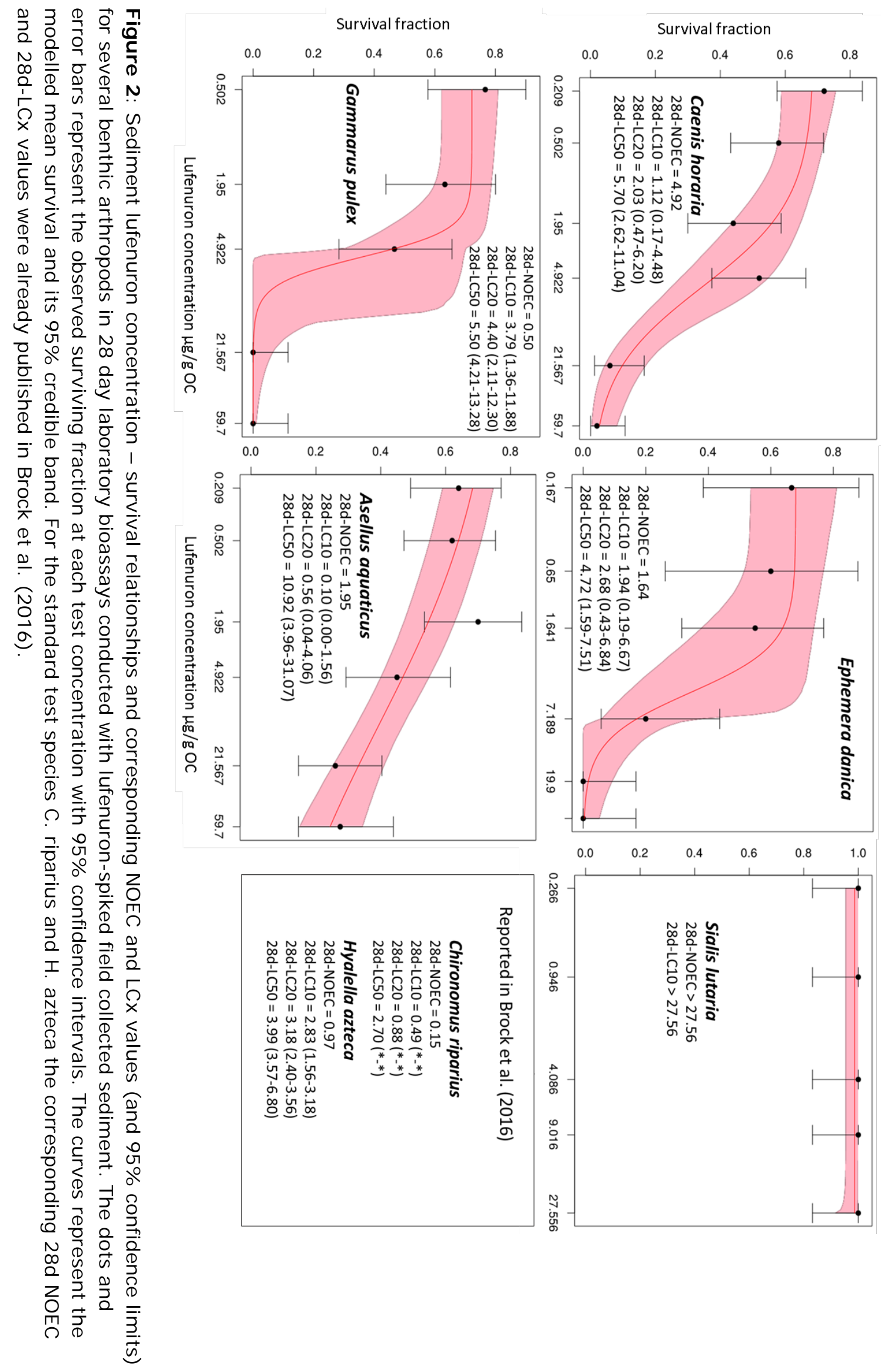



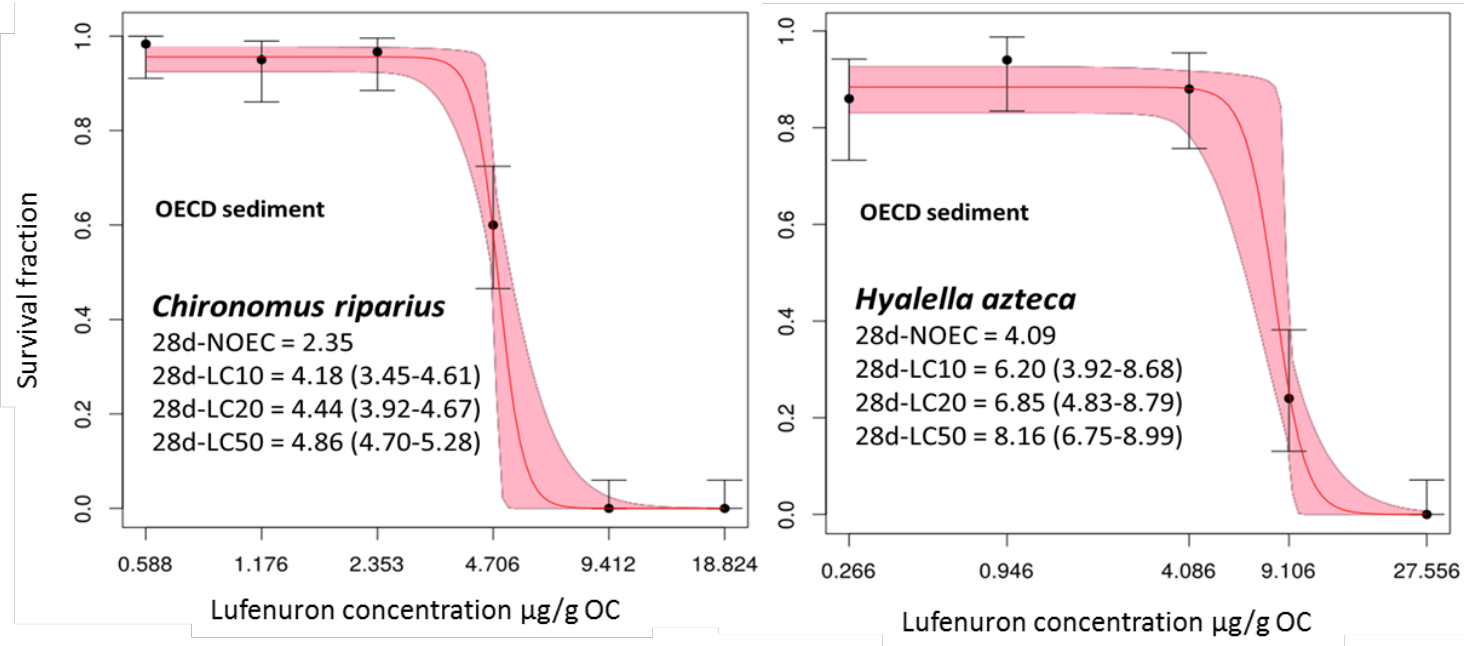

Figure 3: Sediment lufenuron concentration - survival relationships and corresponding NOEC and LCX values (and 95\% confidence limits) for the standard benthic test species C. riparius and $\mathrm{H}$. azteca in 28 day laboratory bioassays conducted with lufenuron-spiked artificial (OECD) sediment. The dots and vertical error bars represent the observed surviving fraction at each test concentration with 95\% confidence intervals. The curves represent the modelled mean survival and its 95\% credible band. The basic data for C. riparius were kindly provided by Syngenta 
SSD 10d-LC50 values

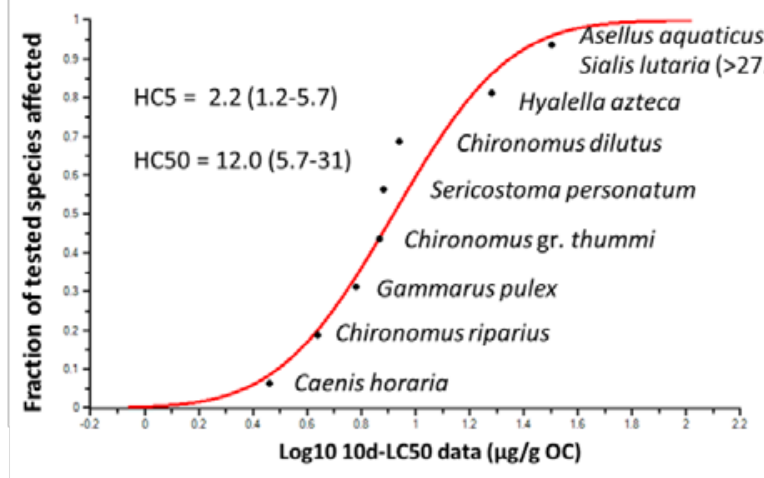

SSD $28 \mathrm{~d}-$-LC50 values

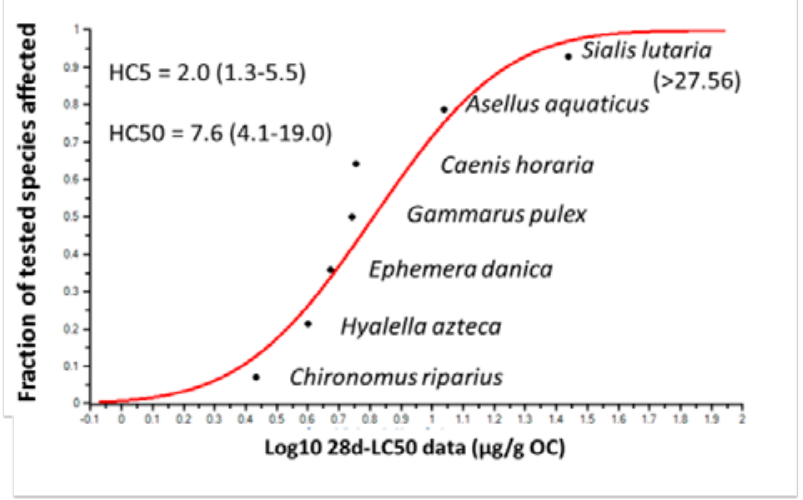

SSD 28d-NOEC values

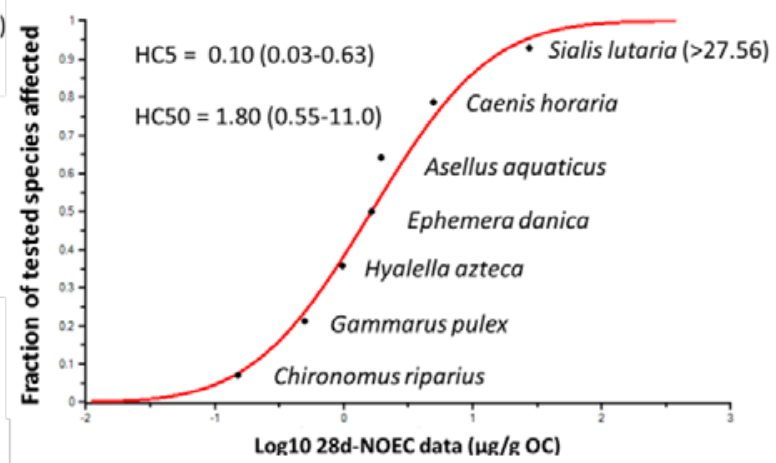

SSD 28d-LC10 values

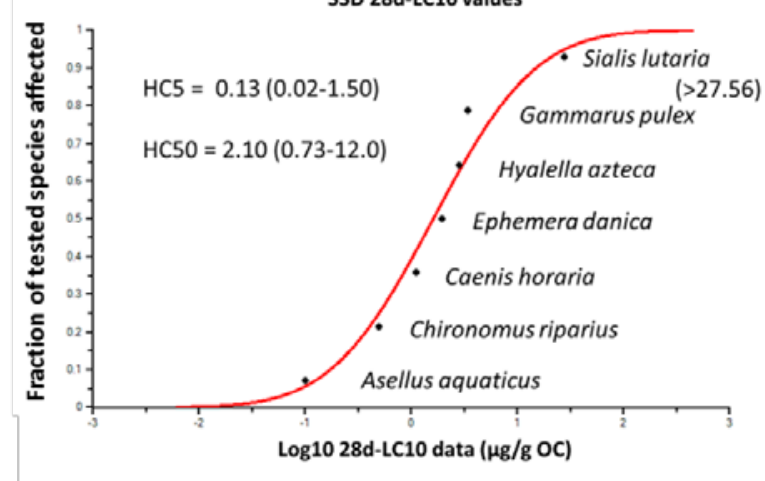

Figure 4: Species sensitivity distributions (SSDs) constructed with 28d-LC10, 28d-NOEC, $28 \mathrm{~d}-$ LC50 and 10d-LC50 values derived from the sediment-spiked laboratory bioassays with the insecticide lufenuron. The Hazardous Concentrations to $5 \%(=\mathrm{HC} 5)$ and $50 \%$ ( = HC50) of the tested species and their 95\% confidence intervals are calculated using the log-normal fitted distribution according to MOSAICSSD. 


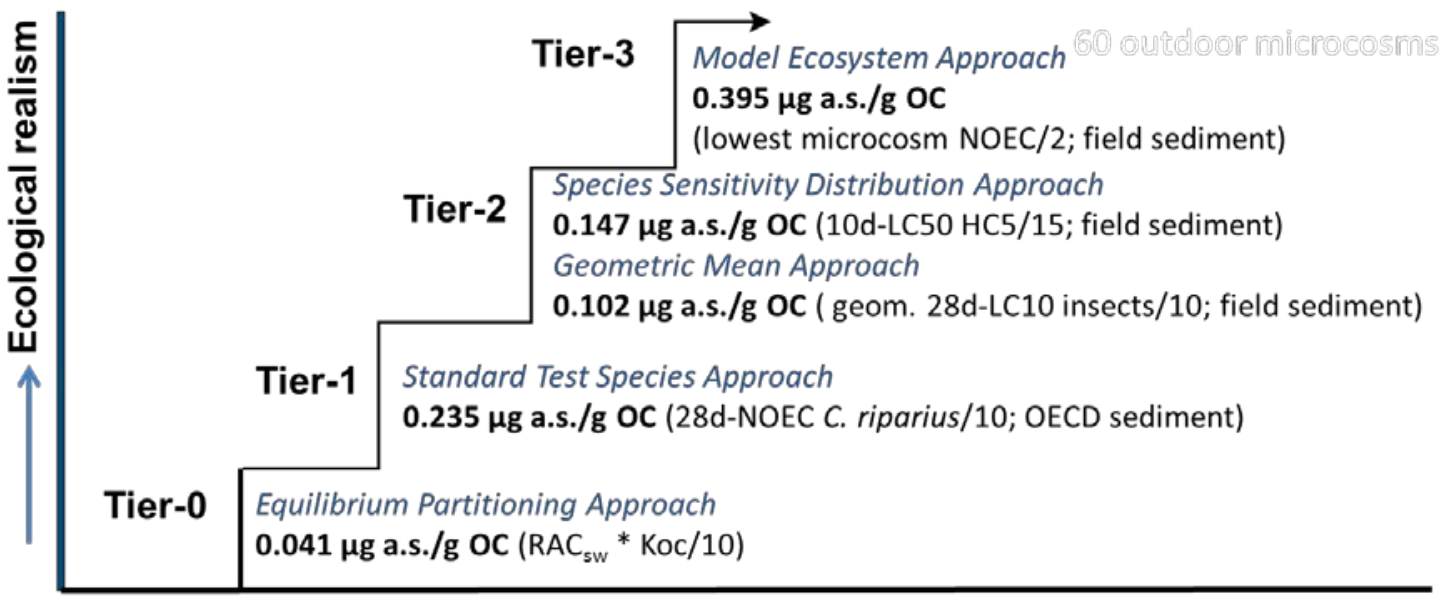

Complexity (experimental data)

Figure 5: Overview of possible Regulatory Acceptable Concentrations for sediment-dwelling organisms ( $R A C_{s e d}$ ) and sediment exposure to lufenuron derived for several effect assessment tiers according to methods described in EFSA (2015). 


\begin{tabular}{|c|c|c|c|c|c|c|c|c|c|}
\hline 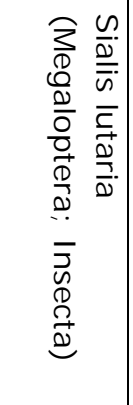 & 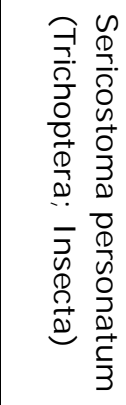 & 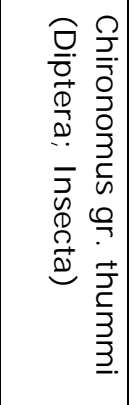 & 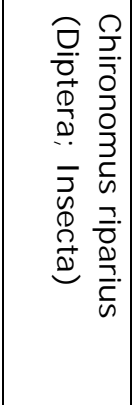 & 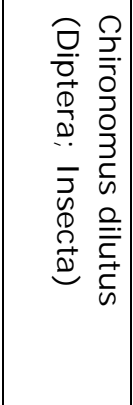 & 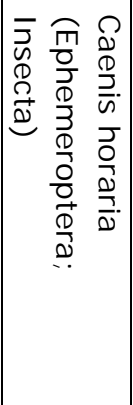 & 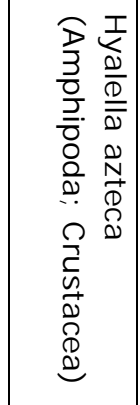 & 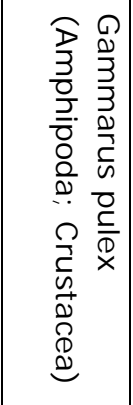 & 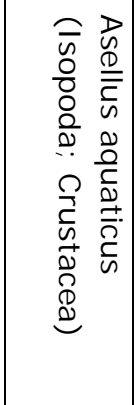 & $\begin{array}{l}-1 \\
\mathbf{y} \\
\underline{\underline{x}} \\
\underline{\underline{\partial}}\end{array}$ \\
\hline 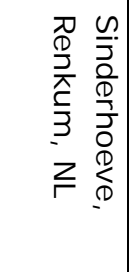 & 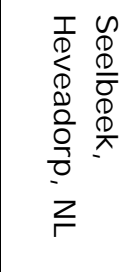 & 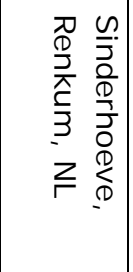 & 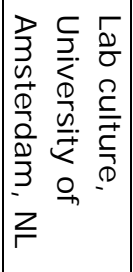 & 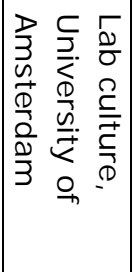 & 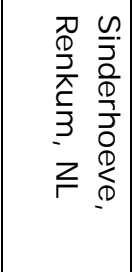 & 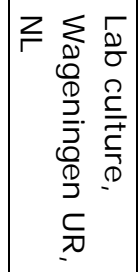 & 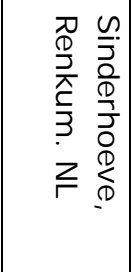 & 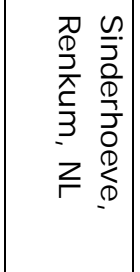 & 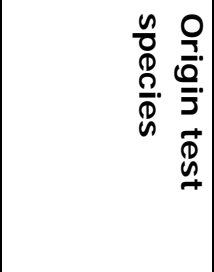 \\
\hline 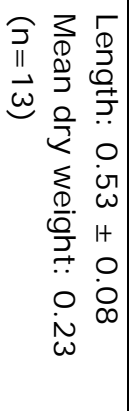 & 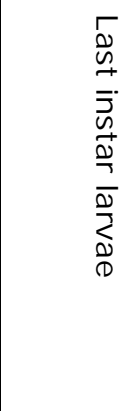 & 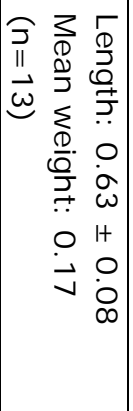 & 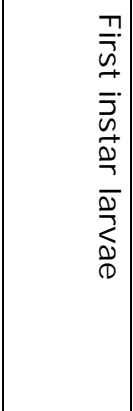 & 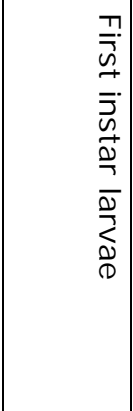 & 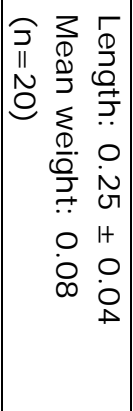 & 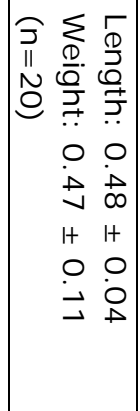 & 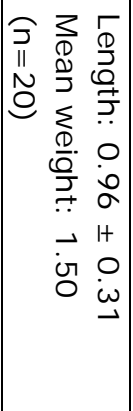 & 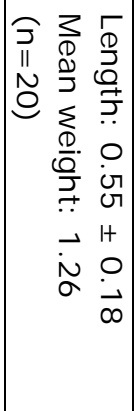 & 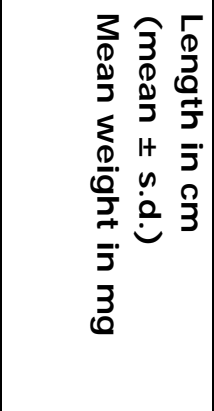 \\
\hline 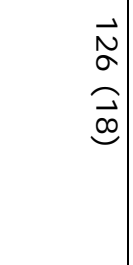 & $\begin{array}{l}\tilde{\omega} \\
\underline{\underline{v}}\end{array}$ & $\begin{array}{c}\tilde{D} \\
\stackrel{\Xi}{\omega}\end{array}$ & $\begin{array}{l}w \\
\tilde{v} \\
\vec{v}\end{array}$ & $\begin{array}{l}N \\
\infty \\
\equiv\end{array}$ & $\begin{array}{l}\stackrel{\sim}{\sim} \\
\stackrel{\omega}{\sigma}\end{array}$ & $\begin{array}{c}\tilde{D} \\
\underline{\omega}\end{array}$ & $\begin{array}{c}\tilde{D} \\
\underline{\omega}\end{array}$ & $\begin{array}{c}\tilde{\Xi} \\
\stackrel{\omega}{\omega}\end{array}$ & 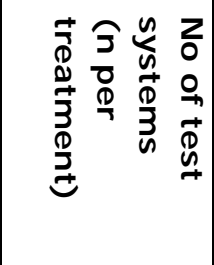 \\
\hline $\begin{array}{l}\text { ज } \\
\text { ò } \\
\text { o }\end{array}$ & 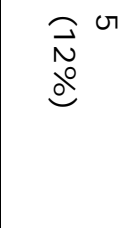 & $\begin{array}{l}\widehat{w} \bullet \\
\dot{w} \\
\stackrel{0}{0} \\
o\end{array}$ & 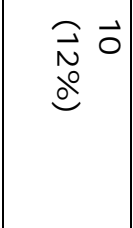 & $\begin{array}{l}\text { 点 } \\
\text { un } \\
\text { o }\end{array}$ & 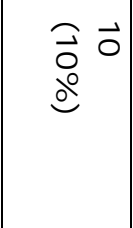 & $\begin{array}{l}\widehat{w} \\
\dot{w} \\
\dot{0} \\
0\end{array}$ & 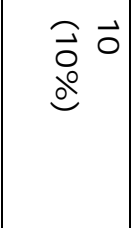 & $\begin{array}{l}\partial^{\circ} \circ \\
\stackrel{\circ}{0}^{\circ}\end{array}$ & 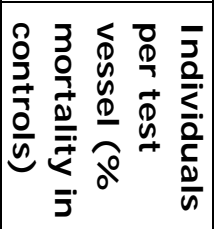 \\
\hline 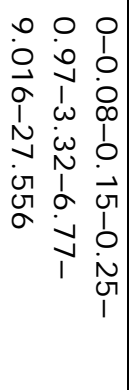 & 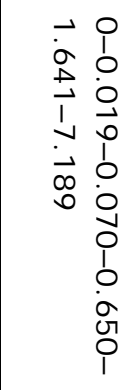 & 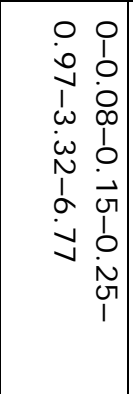 & 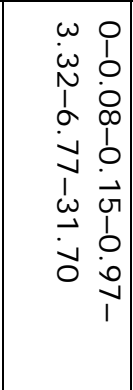 & $\begin{array}{ll}0 & 0 \\
0 & 1 \\
0 & 0 \\
N & 0 \\
1 & 0 \\
N & 0 \\
1 & 1 \\
u & 0 \\
0 & i \\
1 & 0 \\
ن & 1 \\
0 & 1 \\
0 & 0 \\
0 & 0 \\
0 & 0 \\
0 & 1\end{array}$ & 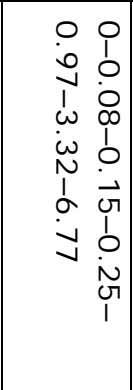 & 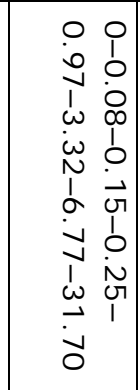 & 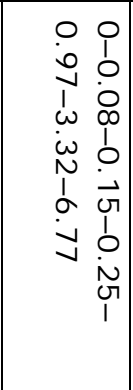 & 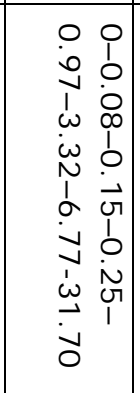 & 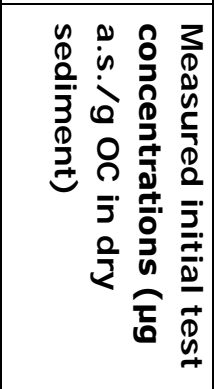 \\
\hline 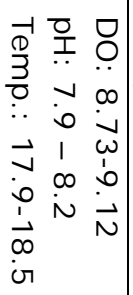 & 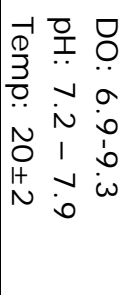 & 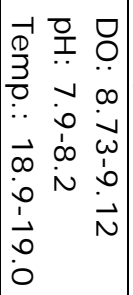 & 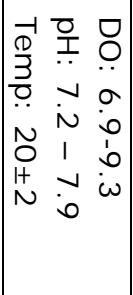 & 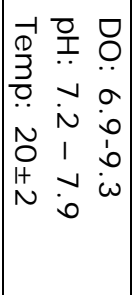 & 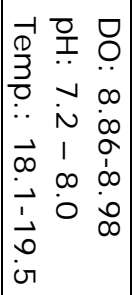 & 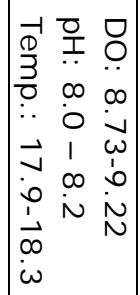 & 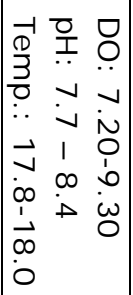 & 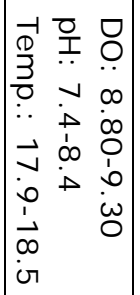 & 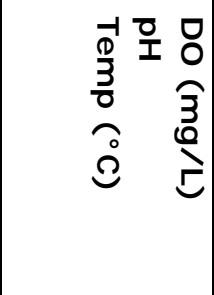 \\
\hline
\end{tabular}




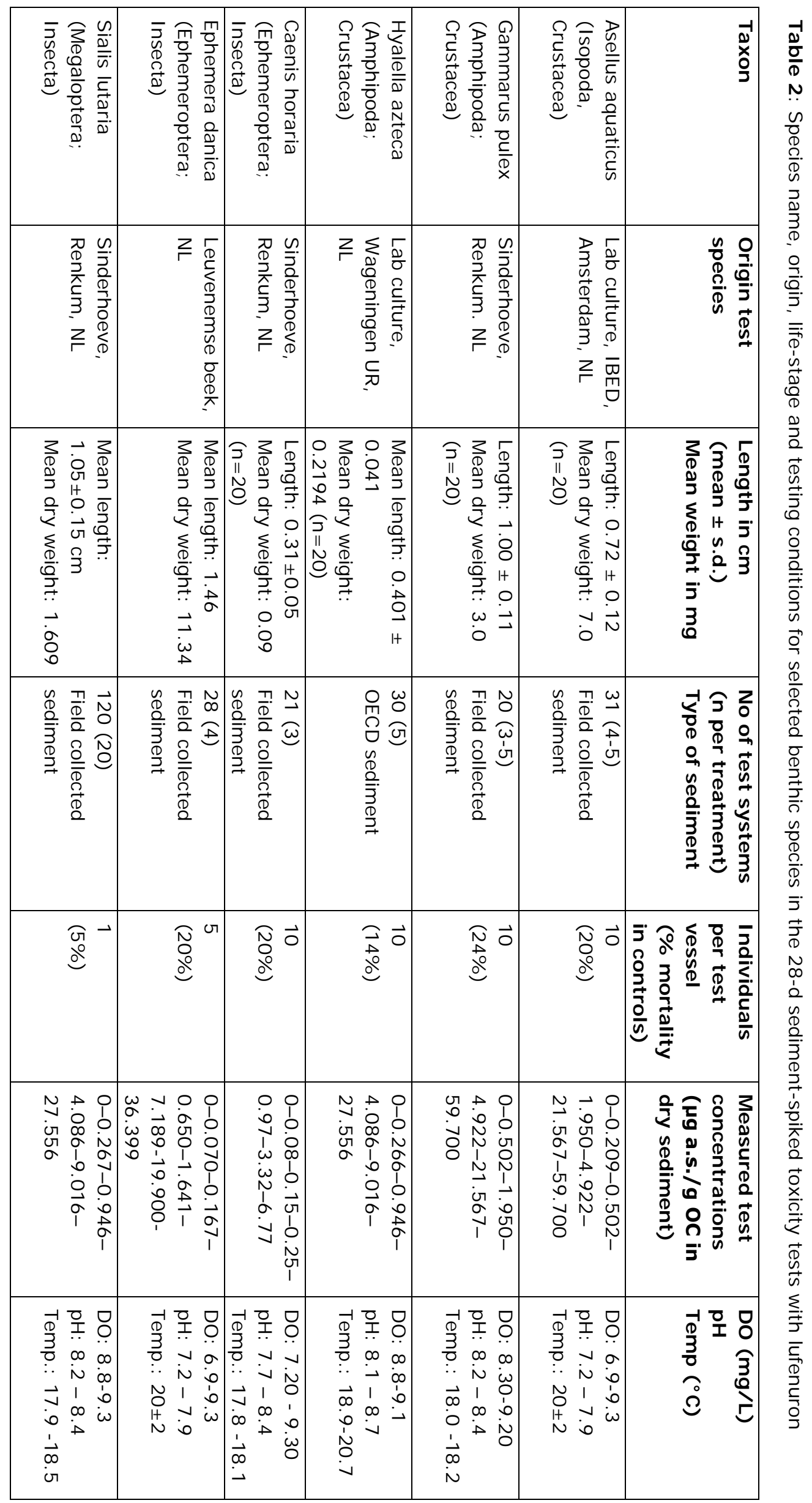

